\title{
La place des littératures africaines dans les collections de la Bibliothèque nationale de France
}

Jean-Marie Compte

\section{(2) OpenEdition}

1 Journals

Édition électronique

URL : http://journals.openedition.org/actesbranly/497

DOI : $10.4000 /$ actesbranly.497

ISSN : 2105-2735

Éditeur

Musée du quai Branly Jacques Chirac

Référence électronique

Jean-Marie Compte, «La place des littératures africaines dans les collections de la Bibliothèque nationale de France », Les actes de colloques du musée du quai Branly Jacques Chirac [En ligne], 3 | 2011 mis en ligne le 21 avril 2011, consulté le 07 septembre 2020. URL : http://journals.openedition.org/ actesbranly/497 ; DOI : https://doi.org/10.4000/actesbranly.497

Ce document a été généré automatiquement le 7 septembre 2020

(c) Tous droits réservés 


\title{
La place des littératures africaines dans les collections de la Bibliothèque nationale de France
}

\author{
Jean-Marie Compte
}

1 Bonjour. Je me réjouis beaucoup de vous présenter une communication dans ce musée du quai Branly qui fait une place si particulière et si importante au livre. On peut dire que c'est un cas assez unique dans les musées de France de mise en avant du livre et de la littérature. Je m'en réjouis beaucoup, de même que je remercie Yves Le Fur qui a su souligner l'importance de la collaboration entre le musée du quai Branly et la Bibliothèque nationale de France dans l'organisation de ce colloque. Comme cela a été rappelé hier, la Bibliothèque nationale de France a une vocation encyclopédique, une mission de conservation et de valorisation du patrimoine écrit et documentaire du plus ancien au plus contemporain. Et c'est précisément cette mission et cette vocation qui rendent complexes la compréhension et l'appréhension de la place des littératures africaines dans les collections, sujet qui m'a été proposé de traiter ce matin et que j'ai accepté volontiers de faire, même si j'ai davantage une approche de généraliste que de spécialiste de ces questions.

2 C'est précisément dans le cadre d'une politique documentaire d'une grande bibliothèque nationale, qu'il est intéressant de comprendre comment une catégorie de littérature noire ou de littérature africaine peut être traitée en la regardant d'un point de vue historique du traitement documentaire au quotidien, ce que je vais m'efforcer de faire maintenant.

3 Les collections de la BNF se sont constituées au fil du temps depuis le $\mathrm{XV}^{\mathrm{e}}$ siècle. Leur longue histoire est précisément à l'origine de leur richesse et elle explique aussi leur immense diversité. On peut dire que cette bibliothèque, par le volume de ses collections, est la première de France et l'une des toutes premières au monde. Ces collections sont réparties sur plusieurs sites: collections spécialisées conservées sur le site de Richelieu au cœur de Paris et - aujourd'hui - les collections proposées depuis quelques années sur le site de Tolbiac, sont gérées de manière thématique par cinq 
départements. Elles sont proposées au public selon deux moyens d'accès, à travers une bibliothèque de recherche située au rez-de-jardin du site François Mitterrand, accessible pour des chercheurs, et une bibliothèque d'étude accessible à partir de 16 ans où l'ensemble des collections est présenté en libre accès dans un certain nombre de salles ; mais bon ce n'est pas le sujet ici, je précise parce que j'aurai bien sûr à revenir sur la façon dont sont utilisées les collections chaque fois qu'on veut entrer dans une discipline où une catégorie particulière.

4 Le principe de l'encyclopédisme suppose que les ouvrages et les documents qui entrent à la Bibliothèque nationale de France, principalement par la voie du dépôt légal qui est le miroir de la production éditoriale nationale, ainsi que de son originalité et ses faiblesses, soient complétés par des acquisitions dans les domaines qu'ils couvrent peu ou mal. On sait que c'est le dépôt légal qui a fortement présidé aux enrichissements de la Bibliothèque nationale depuis son origine jusqu'aux années 1920. Puis il y a eu une période jusqu'aux années 1970-1980 pendant lesquelles il y a eu une gestion plus aléatoire. Je vais y revenir.

D'autre part, si la Bibliothèque nationale n'a pas dans ses domaines de compétence d'établissements spécialisés à concurrencer, elle reste un lieu unique où se trouvent rassemblées des ressources de niveau élevé dans tous les domaines et dans toutes les disciplines. On pourra reparler plus tard, si vous voulez, de la question de l'interdisciplinarité.

6 Ce qui caractérise la place des littératures africaines dans les bibliothèques nationales de France, c'est une logique documentaire qui a su finalement s'adapter au fil du temps. C'est aussi une volonté de signalement de ces collections pour les faire ressortir de ce qu'on pourrait appeler le "magma du catalogue central", ou même de certains catalogues spécialisés particulièrement difficiles d'accès. S'est enfin plus récemment affirmée la définition d'une politique documentaire avec des objectifs clairement définis, revendiqués et évalués dans la réalisation.

7 Jusqu'à la fin de la période coloniale, l'excellent fonctionnement du dépôt légal assuré dans les départements français d'Afrique a permis que les collections de périodiques: revues, journaux, monographies, brochures, livres imprimés ont fait de cette collection de la bibliothèque un des plus beaux fonds de la littérature africaine. On n'en a pas sur le moment vraiment conscience et cela n'est mesurable que parce qu'un travail à la fois de recherche et de bibliographie extrêmement minutieux et précis a été mené, un travail dont vous pouvez lire le résultat dans deux catalogues. L'un est publié en 1984 : « littérature africaine à la Bibliothèque nationale, catalogue des ouvrages d'écrivains africains et de la littérature critique s'y rapportant, entrés à la Bibliothèque nationale entre 1973 et $1983 »$. Le second est publié en 1991 avec un intitulé un peu voisin: « littérature africaine à la Bibliothèque nationale, catalogue des ouvrages africains et de la littérature critique s'y rapportant " entre les deux (1920 à 1972). Donc ces deux publications sont le fruit du travail mené par Paulette Lordereau. Ils sont le fruit d'une collaboration avec le CNRS. Alain Ricard a suivi aussi de près ce travail en particulier. L'association pour l'étude des littératures africaines y a aussi participé. Bernard Mouralis a prêté la main au bon achèvement de ce travail, ainsi que d'autres personnes peut-être présentes dans la salle aujourd'hui. Pour ces deux catalogues, il y a un classement par pays avec une montée d'exhaustivité, même si quantitativement cela ne traite pas un nombre considérable de volumes. Simplement l'identification d'un certain 
nombre d'ouvrages et d'écrivains africains, le repérage... cela permet aux chercheurs d'y accéder.

8 Actuellement, un autre travail du même type est achevé et il sera publié en ligne cette année. Il s'agit d'une publication préparée par Mwatha Ngalasso qui aura une préface d'Alain Ricard et qui concerne les écrits en langues africaines. Il s'agit du répertoire du fond des anonymes conservés à la Bibliothèque nationale de France avec comme tranche chronologique 1830-1960. Ces trois cents ouvrages ne sont en réalité pas tous anonymes. Cet ensemble est une œuvre très utile parce qu'elle établit une cartographie des langues dans lesquelles ces ouvrages ont été rédigés. Il y en a plus de quatre-vingts. Ce qui montre bien la diversité et la spécificité de la collection telle qu'elle s'est intégrée dans les fonds et dans les différentes parties de la bibliothèque.

En tous les cas, il ressort de ces travaux, tels qu'ils ont été exécutés au cours des trente dernières années, que la littérature africaine en langues européennes occupe une place évidemment prépondérante dans les collections de la Bibliothèque nationale, par rapport aux productions en langues africaines. Jusqu'aux années 1990, en raison de l'organisation des fonds et de la conception que l'on avait alors de la politique documentaire, il fallait pour comprendre la place des littératures africaines réaliser les travaux que j'ai cités. De plus, un certain nombre de monographies ont permis de compléter les lacunes du dépôt légal, de manière à couvrir le domaine en question. Puis, à partir de 1994, a été créée à la Bibliothèque nationale de France une charte documentaire conçue, écrite, rédigée et décidée avec la création du site FrançoisMitterrand de donner un accès différent de celui qui existait jusqu'à présent, en les présentant en accès (comme on l'a dit tout à l'heure) à la fois dans la bibliothèque du haut-de-jardin et la bibliothèque de recherche du rez-de-jardin. Cela a complètement transformé le rapport entre le public (que l'on soit chercheur ou non) et ces collections. Cela a été facilité par l'adoption d'un système de classification qui a permis de montrer et de présenter des littératures africaines en tant que telles avec des distinctions fondées naturellement sur une approche linguistique. On peut dire que depuis lors à la fois l'enrichissement des collections et leur présentation se fait selon une logique de corpus. Au sein de deux services principalement: le département littérature et art au service des littératures étrangères recense les œuvres de près de deux cents écrivains d'Afrique. Leurs œuvres sont présentées à la fois en langue originale, ensuite une traduction de référence, ensuite des essais critiques sur l'œuvre. Vous en avez un aperçu dans la biographie sélective qui est distribuée depuis hier à l'entrée du colloque. Le service de littérature française est le deuxième à mettre en avant la littérature africaine. C'est un peu la même chose autour du corpus, en tout cas cela vaut pour la littérature $\mathrm{du} \mathrm{xx}^{\mathrm{e}}$ siècle. La présentation est un peu différente. Les œuvres complètes lorsqu'elles existent, ensuite les monographies parues séparément et enfin les essais critiques.

Pour le $\mathrm{xxI}^{\mathrm{e}}$ siècle un choix différent a été fait. Il a été décidé de ne pas faire de distinction entre les auteurs français et les auteurs d'expression française. Voilà : ils sont présentés indifféremment. Je pourrais m'en expliquer si vous le voulez tout à l'heure.

11 Je viens de parler des collections de monographies. Pour ce qui est des périodiques, la richesse des collections sur le plan historique est absolument évidente. Cette partie est sans doute plus complexe à aborder car les recoupements n'étaient pas forcément ceux qui se font de manière habituelle et ces documents peuvent être plus difficiles d'accès. 
Davantage que pour les livres, il a été fait un travail sous la forme d'échanges qui a été très opérationnel jusqu'à la période des indépendances et qui aujourd'hui bute sur toutes les difficultés que connaissent les éditeurs, les bibliothèques dans les pays d'Afrique. On ne peut que le déplorer.

Pour rendre compte de la diversité des collections de littérature africaine il faut s'intéresser aux évolutions survenues dans une période plus récente et qui sont extrêmement importantes en termes de diffusion, de connaissances et sans doute aussi pour la recherche.

L'intégration récente, au sein de la Bibliothèque nationale de France du centre national de la littérature pour la jeunesse, que certains connaissent parmi nous sous le nom de "la joie par les livres ", a permis aux collections de s'accroître d'un fonds unique de livres pour la jeunesse qui est riche de sept cents volumes publiés dans les pays d'Afrique subsaharienne, dans l'océan Indien excepté La Réunion. Ils sont principalement en français, en entrent aussi dans d'autres langues, africaines comme par exemple en bambara, en wolof, en puulaar, en kiswahili, etc. Certains d'entre eux, il faut le signaler, sont bilingues. Il faut ajouter à cet ensemble qui est d'une richesse extraordinaire les très nombreux livres pour la jeunesse publiée par des auteurs, illustrateurs africains francophones en France, en Belgique, en Suisse et qui figure au même titre dans ces collections. Donc voilà sans doute des pistes nouvelles pour la recherche à partir de l'exploration qui peut en être faite.

Vous connaissez peut-être les signets de la bibliothèque nationale de France qui accordent une place importante aux littératures africaines à travers donc le recensement de sites web, d'écrivains, de sites privilégiés... peut-être davantage une approche en termes de "Cultural Studies». S'y ajoutent des sites de bibliographies qui sont vérifiés pour être accessibles en ligne. Pour cette seule catégorie, on en recense près de deux cents... plus ou moins spécialisés, évidemment.

15 Pour terminer, je souhaiterais insister sur le fait qu'aujourd'hui il me semble possible, envisageable et souhaitable d'aller plus loin pour renforcer la place des littératures africaines, des littératures noires à la BNF. Il faudrait peut-être le faire en surmontant ce qui est un problème aujourd'hui d'ordre économique, parce que les crédits notamment d'acquisition de livres - nécessitent des combats budgétaires qui ne sont pas toujours faciles à gagner.

16 L'autre raison me semble nécessiter une approche beaucoup plus coopérative. Il y a ici deux pistes qui s'ouvrent. C'est l'obligation qui nous est faite de proposer la numérisation des collections. C'est un travail dans lequel la Bibliothèque nationale de France s'est lancée depuis la création de Gallica au début des années 1990. Cela s'est beaucoup renforcé grâce aux moyens obtenus pour ce faire. Si vous consultez Gallica, vous vous rendrez compte que la présence des livres d'écrivains africains est là encore considérable. Cela s'explique par la question des droits, puisqu'on a choisi une barrière chronologique pour le respect du droit d'auteur. Néanmoins, le travail tel qu'il a été mené pour la présentation autour de la logique de corpus nous impose de réfléchir en termes de collaborations avec les universités, les bibliothèques spécialisées. Il est tout à fait envisageable de se concerter pour la numérisation et de collaborer. Je pense que dans les prochains mois des annonces pourront être faites en ce sens, notamment en raison des moyens accordés à la Bibliothèque nationale de France au titre du grand emprunt. Un autre champ, c'est tout ce qui relève du partage documentaire parce que le paysage des bibliothèques qui s'intéressent aux littératures africaines est en train 
d'évoluer. Comme vous le savez, à proximité de la Bibliothèque nationale ouvrira en 2011 la bibliothèque universitaire des langues et civilisations. Il existe des collaborations dans le domaine des littératures orientales et nous souhaitons évidemment les étendre aux littératures africaines dès que ce sera possible. Cela passera par de nombreuses collaborations y compris avec le musée du quai Branly.

Pour conclure, je voudrais dire qu'au-delà des questions scientifiques, des questions documentaires qu'elle permet de poser, l'examen de la place des littératures africaines dans une grande bibliothèque nationale comme la BNF est également un sujet qui relève éminemment de la politique culturelle de l'établissement. Pour cette raison, il ne doit plus être aujourd'hui seulement dans le cadre particulier de ce seul établissement, mais aussi dans un cadre plus coopératif. Je vous remercie.

\section{ANNEXES}

\section{Débat}

\section{Romuald Fonkoua (modérateur)}

Je vous remercie pour cette contribution qui permet de toucher du doigt les problèmes que connaissent ceux qui sont obligés de tracer des livres et auquel on doit avoir accès. Vous avez eu l'amabilité de rappeler un travail qu'avait initié Alain Ricard et auquel nous somme un certain nombre à avoir prêté notre petite main. À l'époque cela se passait à la Bibliothèque nationale, à Richelieu, où l'on se retrouvait tous les jeudis après-midi, si mes souvenirs sont exacts. Merci de l'avoir rappelé.

Vous avez posé un problème tout à l'heure et vous avez demandé d'intervenir làdessus. Je vais donc rebondir sur le sujet : c'est la logique d'un corpus qui vous conduit au $\mathrm{Xx}^{\mathrm{e}}$ siècle et au $\mathrm{xxI}^{\mathrm{e}}$ siècle. Alors qu'en est-il ? Pourquoi changez-vous entre le $\mathrm{xx}^{\mathrm{e}}$ et le $\mathrm{xxI}^{\mathrm{e}}$ siècle cette logique du classement des corpus ? C'est ma première question. Seconde question : c'est celle des échanges de périodiques. Vous avez dit quelque chose que je trouve assez juste. C'est que la Bibliothèque nationale a intégrée à un certain nombre de périodiques, en particulier les périodiques africains. Vous avez raison, puisque toutes les annales des universités africaines se retrouvent à la BNF. Après 1975, elles disparaissent. Alors, de deux choses l'une : ou alors c'est plus classé pour l'Afrique ou alors bibliothèque nationale ne fait plus le travail de recueil, ou alors les annales ne sont plus publiées...

\section{Jean-Marie Compte}

Sur la première question, c'est le service des littératures françaises qui est concerné par une présentation différente. Là, je dirais que cela est fait dans une logique de public. On observe comment le public utilise les collections. Il ne s'agit pas de les présenter à personne. L'autre raison, c'est la production éditoriale telle qu'elle est faite. La plupart des auteurs du corpus des écrivains d'expression française publiait chez les grands éditeurs français. D'ailleurs, c'était mentionné lors d'une communication que j'ai 
entendue. On a essayé de coller plutôt à cette réalité-là. Bon, je concède que c'est plutôt une logique bibliothèque qu'une logique culturelle. Cela montre aussi que l'on doit s'adapter au fil du temps.

Pour la seconde question, j'ai peut-être exagéré en paraissant indiquer que le problème des échanges n'était que d'un côté. En réalité on est deux partenaires. Je ne connais pas les raisons précises du cas dont vous parlez. Cela tient aussi à des problèmes je dirais plus circonstanciels. En tout cas, la BNF a fait le choix de ne plus échanger mais d'acquérir des livres que le partenaire à l'étranger souhaite recevoir. Alors cela peut aussi expliquer un certain nombre de problèmes.

Autre chose : pour apporter une réponse qui était posée hier au sujet d'Haïti et de la présence des écrivains haïtiens du XIX ${ }^{e}$ siècle dans les collections de la BNF. Cette présence tout à fait importante, je crois qu'elle reflète aussi ce que disait Mickael Dash du néocolonialisme. Les œuvres de ces écrivains sont conservés au département philosophie, histoire, sciences de l'homme. Un certain nombre de ces livres sont accessibles sur Gallica. Ils ont été numérisés parce que le département philosophie, histoire, science de l'homme a fait le choix de livres qui se trouvent sous cette cote. Les œuvres de ces écrivains sont là, ils sont une vingtaine. Voilà, c'était pour apporter une réponse tardive à une question posée hier.

\section{Questions du public}

Bonjour, je suis Virginie Coulon, l'auteur d'une base de données sur la littérature africaine. Elle se trouve sur le serveur du Centre d'études d'Afrique noire à Bordeaux et qui est le fruit de ce fameux séminaire organisé par Alain Ricard. J'ai une remarque à faire : en faisant l'ITAF, je me suis aperçu que la BNF est malheureusement toujours à la traîne par rapport à la littérature publiée en Afrique. Il y a un champ dans l'ITAF qui s'appelle « localisation » et qui permet de prouver l'existence d'un ouvrage.

Malheureusement, pour ce qui concerne les ouvrages publiés en Afrique il n'y a souvent pas de traces en France, sauf au Celfa à Bordeaux III ou à la Norwestunion University Library. Voilà, c'est ma remarque. La question : hier soir au dîner, j'ai pris connaissance d'un problème dont j'espère que vous êtes au courant. Elle nous a parlé du travail qu'elle est en train de faire sur les carnets d'olivier de Sanderval et elle a bien entendu pu trouver ces carnets dans un format numérisé, mais dans un format qui interdit la recherche. C'est décourageant. Ça pose un problème pour le chercheur. Voilà.

\section{Jean-Marie Compte}

Nous sommes dans un certain nombre de cas obligés, effectivement, de reprendre une numérisation. Je vous invite à le signaler parce que l'on essaie en général de la reprendre de façon prioritaire. D'ailleurs il y a une petite équipe auprès de Gallica qui fait systématiquement ce travail. Mais bon, ce n'est pas toujours possible de reprendre. Mais dans des cas qui sont signalés, cela sera repris. Aujourd'hui, on ne peut pas faire l'impasse sur ce que vous dites. Quand au site que vous mentionnez, il est référencé dans les signets de la BNF. Cela n'évite pas bien sûr pas ce que vous pointez comme problème.

\section{Alain Ricard}

Je voulais vous remercier, Romuald Fonkoua et Jean-Marie compte d'avoir fait allusion à ce travail que nous avons mené dans les années 1980 , mais je voulais simplement dire que c'était un travail militant. Il s'agissait au fond de faire émerger dans le continent 
noir un iceberg africain. Paulette Lordereau, qui était conservateur était également militante de ces questions-là et je crois que c'est très important. Donc : une présence de l'Afrique dans les collections qui existait. Et je voudrais faire une suggestion : vous avez fait allusion au travail et à l'ingéniosité de recherche de Paulette Lordereau qui était fière d'avoir identifié des écrits anonymes dans les fichiers et les manuscrits orientaux. Il s'agit en fait souvent de grammaires de langues africaines qui ont été rédigées au début $d u X X^{e}$ ou à la fin $d u X^{e}{ }^{e}$ et le travail de notre groupe de recherches a consisté à identifier les langues dont il était question aujourd'hui, les systèmes graphiques. Et donc : je crois que cela se prêterait particulièrement bien à une numérisation. Je voulais simplement sa suggestion. On a parlé hier et je voulais tout simplement la refaire en public. Je souhaiterais donner la priorité à la numérisation de ces textes qui dans beaucoup de cas font appel à des alphabets que l'on utilise plus ou que l'on utilise encore, mais rarement. Cela permet de faire apparaitre l'Afrique comme un continent graphique et que cette histoire-là n'est pas que du passé. Merci.

\section{Jean-Marie Compte}

Tout à fait. Et si cela peut être une conséquence de ce colloque j'en serais très heureux et j'inviterai volontiers un certain nombre de collaborateurs, dont je dois dire aussi qu'ils ont participé à la préparation de cette communication en préparant un corpus de ces livres pour pouvoir les numériser sur Gallica. Cela permettra aussi de déboucher sur un travail éditorial sur Gallica.

\section{Questions du public}

Je vous remercie d'avoir soumis le travail mené par « la joie par les livres » dans le cadre de la Bibliothèque nationale de France. «La joie par les livres » qui depuis 20 ans est une fenêtre sur les éditeurs de jeunesse en Afrique et qui permet à travers des partenariats tels l'association internationale des libraires francophones, de diffuser mieux ces ouvrages de jeunesse est donc d'être une bonne opportunité pour les visiteurs de vendre leurs livres également, donc qui a un aspect commercial aussi. Mais puisque vous parlez de perspectives, je voudrais simplement vous poser la question : comment réfléchir demain de manière à ce que « la joie par les livres » puisse s'ouvrir aux éditions non seulement de jeunesse mais également à l'édition plus large. Je pense justement qu'à l'heure où l'édition africaine est en pleine ébullition, il y a des créations d'éditeurs dans tous les pays, il ne faudrait pas que la BNF rate le coche à ce niveau-là. Il ne s'agit pas seulement de rendre accessible les catalogues, mais de rendre également les ouvrages accessibles. Donc la question est : comment rendre une perspective éditoriale comme celle de «La joie par les livres » à l'édition plus largement généraliste.

\section{Jean-Marie Compte}

Je ne voulais pas entrer dans les détails, mais vous connaissez sûrement la revue Takam Tikou (http://takamtikou.bnf.fr/) conçue par le centre national de la littérature pour la jeunesse, précisément en direction de l'international et notamment de l'Afrique. Cette revue va connaitre une nouvelle vie puisqu'à partir du printemps prochain elle sera publiée en ligne - et donc elle pourra aussi servir de chambre d'écho pour partie à ce que vous dites mais bon cela ne suffira pas, peut-être. D'autre part, là encore j'insiste sur les questions de collaborations et de coopération. Il y a ce que la « joie par les livres » peut faire en direction notamment de chercheurs ou d'universitaires qui travaillent sur ces sujets en Afrique. Le prochain numéro, là je parle sous le contrôle du 
collègue du centre national de la littérature pour la jeunesse présent dans la salle, fera mention d'un travail mené au Burkina Faso actuellement. Il s'agit de l'une des premières études exhaustives sur l'édition du livre pour la jeunesse au Burkina Faso. Ce sont des travaux comme ceux-là qui pourront aller dans le sens de ce que vous souhaitez.

\section{AUTEUR}

\section{JEAN-MARIE COMPTE}

Né en 1958, archiviste paléographe, conservateur général des bibliothèques, il est depuis 2008 le directeur du département littérature et art de la Bibliothèque nationale de France. Après ses études à l'Ecole nationale des chartes (1981-1985), il dirige la bibliothèque départementale de la Creuse (1985-1990) puis la Bibliothèque municipale classée de Poitiers (1990-2001). De 2001 à 2004, il est conseiller du directeur de la Bibliothèque d'Alexandrie en Egypte. Il dirige ensuite le Centre national de la bande dessinée et de l'image entre 2005 et 2007. 